\title{
Konflikte um urbane (Un-)Sicherheit - Einführung in das Schwerpunktheft
}

\author{
Peter Bescherer · Tim Lukas
}

(C) Springer Fachmedien Wiesbaden GmbH 2017

\section{Einleitung}

Die Idee zu diesem Heft entstand vor dem Hintergrund eines Unbehagens: Städtisches Leben wird gegenwärtig vor allem unter Aspekten der Bedrohung und Unsicherheit wahrgenommen. In erster Linie aufgrund terroristischer Angriffe, die obwohl statistisch eine verschwindend kleine Wahrscheinlichkeit besteht, ihnen zum Opfer zu fallen - tiefe Spuren in der öffentlichen Diskussion hinterlassen und in unsachgemäßer Weise mit Fragen der Aufnahme und Integration Geflüchteter verknüpft werden. Aber auch im Hinblick auf ,klassische" Kriminalität (Wohnungseinbrüche), Gesundheitsgefährdungen (Feinstaub und Abgase), Naturgefahren (Überschwemmungen und Starkregen) oder politisch motivierte Proteste (,neue Qualität der Hamburger G20-Krawalle) gelten Städte als unsicher. Obwohl selbst als wissenschaftliche Beobachter und Mitarbeiter in anwendungsorientierten Forschungsprojekten in das Handlungsfeld verstrickt, irritiert uns die (nicht ganz so) ,neue Qualität ${ }^{`}$ des Sicherheitsdiskurses. Während die Stadtforschung seit ihren Anfängen die Ambivalenz urbaner Lebensweisen betont und kritische Kommentare immer wieder auf Zusammenhänge und Unterschiede von Kriminalität, Kriminalitätsfurcht und sozialen Ängsten hinweisen, reagieren Politiker*innen, Behörden, aber auch Bürger*innen - es liegt ja auch nahe - mit einem einfachen Rezept: Gegen Unsicherheit hilft nur mehr Sicherheit.

\footnotetext{
P. Bescherer $(\square)$

Internationales Zentrum für Ethik in den Wissenschaften, Eberhard Karls Universität Tübingen, Wilhelmstraße 19, 72074 Tübingen, Deutschland

E-Mail: peter.bescherer@posteo.de

T. Lukas $(\triangle)$

Bevölkerungsschutz, Katastrophenhilfe und Objektsicherheit, Bergische Universität Wuppertal, Gaußstraße 20, 42119 Wuppertal, Deutschland

E-Mail: lukas@uni-wuppertal.de
} 
Natürlich müssen Gewalt unterbunden, Straftaten verfolgt und Anschläge nach Möglichkeit verhindert werden. Aber die ,Beseitigung der Unsicherheit ' ist ein dauerhaft nicht tragfähiger Ansatz. Denn Sicherheit wird sich niemals abschließend herstellen lassen - jedes Stück erreichter Sicherheit wird zur ,Entdeckung ' neuer Unsicherheiten und gesteigerten Bedürfnissen nach ihrer Bewältigung führen. Darüber hinaus ist die ,Herstellung ' von Sicherheit, und darauf zielt der Titel des Heftes, ein von Gegensätzen und Konflikten bestimmter Prozess. Denn Sicherheit ist eben „nicht irgendein hohes oder rätselhaftes Gut, dem jede und jeder gern zustimmen mag, um das sich alle scharen und das es stets - und um jeden Preis - zu erreichen gilt“ (Eick 2014, S. 154). Was die einen als wünschenswerte Sicherheit ansehen, kann die Sicherheit der anderen gerade einschränken; wer die Diskussionen um Trinker und Suchtkranke in den Innenstädten verfolgt, weiß wovon die Rede ist. Und selbst mit der Perspektive auf ,Herstellung ' und ,Verteilung ' - die die anwendungsorientierte Forschung kaum vermeiden kann - werden Konflikte nicht ausbleiben, sind die sicherheitsrelevanten Ressourcen (Geld, Arbeit, Aufmerksamkeit) doch grundsätzlich begrenzt.

Konflikte um urbane Sicherheit bestehen damit in mindestens drei Hinsichten: Erstens bezüglich der Wahrnehmung und Aufmerksamkeit, die darüber entscheiden, was überhaupt als ,Sicherheitsrisiko" gilt und was nicht. Zweitens sind Konflikte im Bereich der politischen Regulierung und Umsetzung von Sicherheitsmaßnahmen zu beobachten, in dem bestimmte Sicherheitsinteressen - oder Interessen mit Berufung auf Sicherheit - durchgesetzt und andere (Sicherheits-)Interessen vernachlässigt werden. Drittens ist die wissenschaftliche Deutung dieser Konflikte selbst kontrovers und von Konflikten durchzogen; Auseinandersetzungen drehen sich etwa um die Plausibilität des Broken Windows-Theorems oder das Zustandekommen von Nachbarschaftsvertrauen und seine Bedeutung für das Sicherheitsgefühl im Quartier.

Die verschiedenen Beiträge im vorliegenden Heft gehen diesen Konflikten nach. Sie spannen einen Bogen von der Betrachtung quartiersbezogener Wahrnehmung und Verarbeitung von Unsicherheit über die Diskussion des polizeilichen Agierens hin zur Rolle der subjektiven Perspektive auf Sicherheit, die einerseits unterrepräsentiert und andererseits selbst bedenkenswert volatil und ambivalent ist. Auch methodisch gehen die Beiträge sehr unterschiedlich vor. Sie illustrieren damit einen potenziellen Konflikt eigener Art, sind doch die evidenzbasierten, auf die Sicherheitslage zielenden Ansätze mit einer an Sicherheitsdiskursen und -dispositiven orientierten Gouvernementalitätsforschung nur begrenzt vereinbar.

\section{Die Beiträge im vorliegenden Heft}

Als einen Aspekt urbaner Sicherheit machen Jan Abt und Holger Floeting die Lebensqualität im Wohnumfeld zum Ausgangspunkt ihres Beitrags. In der Wahrnehmung von Sicherheit und Unsicherheit sind die sozialräumlichen Bedingungen des Quartiers eine zentrale Größe der Kriminalprävention, die baulich-gestalterische ebenso wie soziale und regulatorische Elemente umfasst. Für die Gewährleistung von Sicherheit und das furchtlose Zusammenleben der Menschen in der Stadt sind daher nicht allein die ,üblichen Verdächtigen“ aus Polizei und Kommune, sondern 
auch private Akteur*innen wie etwa die Wohnungswirtschaft zuständig. Anhand von drei Fallstudien zeigen Abt und Floeting wie unterschiedlich die Akteursnetzwerke der Kriminalprävention in den untersuchten Städten sind, wobei sich die Rollen der einzelnen Akteur*innen als abhängig von eigenen Schwerpunktsetzungen, der Verfügbarkeit von Personal und den jeweils prägenden Kooperationskulturen innerhalb der Städte erweisen. Zwar sehen die Autoren angesichts struktureller Lücken (,Structural Holes“, vgl. Burt 1992) in den Akteursnetzwerken durchaus Optimierungspotenziale für die Zusammenarbeit, sie warnen jedoch davor, die Beschäftigung mit Sicherheit im Quartier unangemessen stark auf Bereiche der (Un-)Sicherheit und Kriminalität zu fokussieren, die sich im öffentlichen Raum zeigen (könnten). Vielmehr geht es ihnen darum, eine Sicherheitskultur der urbanen Unsicherheit zu ermöglichen, in der eben nicht alles als störend Empfundene aus städtischen Räumen entfernt wird.

Die umgehende Beseitigung auch kleinster Ordnungsstörungen postuliert bekanntlich der Broken Windows-Ansatz, der erstmals 1982 von George L. Kelling und James Q. Wilson veröffentlicht wurde (Kelling and Wilson 1982) und heutzutage zum alltagskriminologischen Standardinventar staatlicher wie kommunaler Akteur*innen im Feld der Kriminalitätsvorbeugung zählt. Hohe Bedeutung für die Verursachung subjektiver Unsicherheitsgefühle wird darin den sogenannten Incivilities zugeschrieben, die als abweichende Handlungen unterhalb der Strafbarkeitsschwelle (z. B. Betteln, Obdachlosigkeit) und als physisch-materielle Erscheinungen der Verwahrlosung öffentlicher Räume (z. B. durch Müll oder Graffiti) subjektiv wahrgenommene Störungen der sozialen und normativen Ordnung signalisieren. Stark kritisiert wurde der Broken Windows-Ansatz nicht allein aufgrund der aus ihm abgeleiteten Strategie des Zero Tolerance Policing, sondern auch wegen der lediglich schwachen empirischen Bestätigung des Zusammenhangs von sozialer Unordnung und Kriminalitätsfurcht. Dies ist der Ausgangspunkt des Beitrags von Dietrich Oberwittler, Heleen Janssen und Dominik Gerstner, die auf der Basis einer Bewohner*innenbefragung und unabhängiger systematischer sozialer Beobachtungen in zwei deutschen Großstädten die furchterzeugenden Wirkungen von urbaner Unordnung nur partiell bestätigen können. Vielmehr zeigt sich, dass die Wahrnehmung sowohl von Unordnung als auch von Unsicherheit maßgeblich vom Anteil sichtbarer Minderheiten angetrieben wird und dieser Effekt sehr stark von den individuellen Einstellungen der Bewohner*innen zu Migration abhängt. Eine positive Einstellung gegenüber Migration schwächt die furchtsteigernden Effekte ethnischer Diversität deutlich ab, während ablehnende und xenophobe Einstellungen sie verstärken.

Die Auswirkungen ethnischer Diversität auf das soziale Miteinander sind spätestens seit den Forschungen von Robert D. Putnam Bestandteil kontroverser sozialwissenschaftlicher Debatten, die unter anderem auch die Effekte auf das subjektive Sicherheitsgefühl in urbanen Nachbarschaften thematisieren. Putnam (2007) hatte auf der Grundlage einer empirisch-vergleichenden Studie US-amerikanischer Stadtviertel einen negativen Zusammenhang zwischen der kulturellen Vielfalt und dem kollektiven Sozialkapital von Wohngebieten behauptet und damit auf die potenziell negativen Konsequenzen von Immigration für die soziale Kohäsion in Einwanderungsgesellschaften hingewiesen. Ausgehend von der Heterogenität der in der Folge ermittelten empirischen Resultate unterzieht der Beitrag von Birte Gundelach den 
kontroversen Forschungsstand einer kritischen Evaluation, wobei die Autorin die Effekte ethnisch-kultureller Diversität auf das soziale Vertrauen als einem zentralen Element des Sozialkapitals in den Mittelpunkt ihrer Analyse stellt. Sie kommt zu dem Schluss, dass nur die Hälfte der empirischen Analysen zum Einfluss ethnischer Diversität auf soziales Vertrauen von einem negativen Zusammenhang berichtet. Generell ist festzuhalten, dass eine Mehrheit der betrachteten Analysen keine robusten Belege für einen kausalen Einfluss ethnischer Diversität auf soziales Vertrauen bietet, da eine genaue Untersuchung möglicher Selektionsprozesse und die Verwendung von Längsschnittdaten bislang zu wenig Beachtung in der Forschung finden. Darüber hinaus stellen verschiedene Studienergebnisse die Universalität eines negativen Zusammenhangs von Diversität und Vertrauen in Frage, weil dieser überhaupt nur in ethnisch besonders stark segregierten Kontexten prävalent ist.

Der Vertrauensbegriff steht auch im Zentrum des Beitrags von Meike Hecker und Jan Starcke, die Zusammenhänge zweier unterschiedlicher Modi des Vertrauens empirisch untersuchen. Ausgehend von einer Bevölkerungsbefragung in zwei deutschen Großstädten analysieren sie, welche Wechselwirkungen zwischen dem Vertrauen in die Polizei und dem sozialen Vertrauen in Nachbarschaften bestehen. In Anlehnung an die von Tom R. Tyler (1990) und Robert J. Sampson (2012) entwickelten theoretischen Ansätze der Procedural Justice und Collective Efficacy stellen sie Polizeiund Nachbarschaftsvertrauen als mehrdimensionale Konzepte dar. Kollektive Wirksamkeit bedeutet dabei zum einen, dass die Bewohner*innen einer Nachbarschaft ein gemeinsames Werteverständnis teilen und wechselseitiges Vertrauen aufbringen und zum anderen, dass sie bereit sind, soziale Kontrolle über ihr Wohnumfeld auszuüben. Hinsichtlich des Polizeivertrauens ist zwischen einem instrumentellen Vertrauen in die polizeiliche Effektivität und einem normativen Vertrauen in die polizeiliche Fairness zu unterscheiden. Im Rahmen ihres Beitrags können die Autor*innen einen positiven Einfluss des Vertrauens in die polizeiliche Fairness auf das Nachbarschaftsvertrauen und die soziale Kontrolle feststellen, während das Effektivitätsvertrauen demgegenüber nur auf das Nachbarschaftsvertrauen Wirkungen zeigt. Hecker und Starcke deuten ihre Befunde dahingehend, dass das Vertrauen darauf, dass die Polizei verbindliche Regelungen durchsetzt und Kriminalität vorbeugt, den Aufbau von Vertrauen in der Nachbarschaft erleichtert und faire Polizeiarbeit das Zugehörigkeitsgefühl zu einer Gemeinschaft stärkt.

Die Bewertung der polizeilichen Effektivität und Fairness wird in erheblichem Maße durch erlebte oder durch von Dritten berichtete Erfahrungen mit der Polizei determiniert. Bereits einzelne Negativerfahrungen mit Polizeibehörden können einen bedeutenden Einfluss auf das Vertrauen in die Polizei ausüben. Die von Sophie Perthus und Bernd Belina berichteten Ereignisse aus Sachsen regen vor diesem Hintergrund zum Nachdenken an. Ausgehend von der von Stuart Hall et al. (1978) vorgelegten klassischen Studie Policing the Crisis deuten sie die polizeilichen und politischen Reaktionen auf die rassistische Hetzjagd von Rechten auf Geflüchtete im sächsischen Bautzen als eine Moralpanik, die Ausdruck einer tieferliegenden Krise der sächsischen Hegemonie sei. Auf der Basis einer umfangreichen Sichtung von Medienberichten und politischen Stellungnahmen sowie leitfadengestützter Interviews mit Vertreter*innen der Zivilgesellschaft in Bautzen zeichnen sie die sukzessive Ethnisierung des Konfliktes und die Kriminalisierung der Geflüchteten 
nach. Polizei, Politik und Verwaltung, aber auch Teile der antirassistischen Zivilgesellschaft übernehmen dabei die Rolle der primären Definierer, deren Deutungen in die Berichterstattung der Medien als sekundäre Definierer einfließen. Als ein Muster weisen Perthus und Belina nach, dass frühe lokale Berichte und späte nationale Hintergrundberichte zwar die Rolle der Rechten thematisieren und teilweise sogar fokussieren, die reichweitenstärksten sekundären Definierer jedoch die Schuld der Geflüchteten an der Hetzjagd in den Vordergrund rücken. Die Täter-Opfer-Umkehr im Bautzener Fall gewinnt bundesweite Bedeutung, da in einer Phase, in der von Seiten der Bundesregierung ,kriminelle Ausländer' wieder offensiver adressiert werden, mit der sächsischen Polizei eine primäre Definitionsinstanz auf den Plan tritt, die der Kriminalisierung Geflüchteter in nationalen Diskursen eine qua Amt einflussreiche Legitimation gibt. ${ }^{1}$

Die „Definitionsmacht der Polizei“ (Feest und Blankenburg 1972) in konkreten Situationen macht die Polizei zu einer intermediären Instanz, die zwischen Gesetzen und realen Vorkommnissen, zwischen dem Staat und dem Alltag der Staatsbürger*innen vermittelt. Basierend auf Henri Lefebvres (1991) Verständnis von Raum als sozialem Produkt und dessen Konzeptualisierung des Urbanen als gesellschaftliche Vermittlungsinstanz bereichert der Beitrag von Anja Feth das vorliegende Schwerpunktheft um eine internationale Perspektive. Am Beispiel von Buenos Aires untersucht die Autorin den Prozess der staatlich-urbanen Raumproduktion durch alltägliche polizeiliche Praktiken. Zunächst veranschaulicht sie, wie Polizist*innen vorgehen, wenn sie ,in Bewegung', d.h. im Stadtgebiet unterwegs sind. Anhand der Präventivfestnahme und des Zugriffs auf die Straßenprostitution als ausgewählte Praktiken der polizeilichen Raumkontrolle, dokumentiert sie, dass die argentinische Bundespolizei zwar massiv auf repressiv-territoriale Maßnahmen setzt, gegen Zahlung von Bestechungsgeldern jedoch regelmäßig darauf verzichtet, rechtswidrige Aneignungen des urbanen Raums zu unterbinden. Feth zeigt, dass es sich bei der polizeilichen Raumproduktion um einen konflikthaften, selektiven, informellen und in sich widersprüchlichen Prozess handelt, in dessen Rahmen die Polizei institutionellen, politischen und ökonomischen Interessen folgt. Deutlich wird in ihrem Beitrag, dass die räumlichen Praktiken der Polizei in Buenos Aires (und nicht nur dort) weniger darauf zielen, die öffentliche Ordnung aufrechtzuerhalten und Kriminalität zu bekämpfen, sondern sich vielmehr aus dem Bemühen ergeben, sich gegenüber Politik und Öffentlichkeit als effektiv im Kampf gegen städtische ,Unsicherheit ' darzustellen - auf Kosten der Rechte benachteiligter oder marginalisierter Bevölkerungsgruppen.

Daniela Krüger, Martin Voss und Kristina Seidelsohn haben marginalisierte Bürger*innen und professionelle Sicherheitsexpert*innen in zwei deutschen Großstädten zu Sicherheitsplanungen auf Basis objektivierter Faktoren und subjektiver Sicherheitswahrnehmungen befragt. Sie erweitern mit ihrem Beitrag das Feld der Sicherheitsakteur*innen um die Perspektive der Feuerwehr und lenken den Blick

\footnotetext{
1 Als vergleichbar stellt sich die Kommunikation der Polizei Aalen dar, die für die jüngsten Ereignisse rund um das Volksfest im schwäbischen Schorndorf zunächst einen Mob von rund 1000 Jugendlichen verantwortlich machte, ,die meisten wohl mit Migrationshintergrund“. Tatsächlich stellten sich die Ereignisse im Nachhinein als weitaus weniger dramatisch dar und Auslöser der Randale waren schließlich ,deutsche Jugendliche“ (Stieber 2017).
} 
auf den Prozess der Sicherheitsarbeit in städtischen (Angst-)Räumen, der - so heterogen die Motivationen und Praktiken der einzelnen Akteur*innen auch sind wesentlich durch Statistiken und Imaginationen geprägt ist. Ausgehend von Michel Foucaults (2004) Gouvernementalitätsansatz und dessen These, wonach die Regierungspraktiken in der Stadt vor allem auf statistischen Daten und daraus berechneten Wahrscheinlichkeiten und Verteilungen beruhen, untersuchen die Autor*innen die Bedeutung und Wirkungen von Statistiken und sozialen Imaginationen als Heuristiken des Sicherheitshandelns. So geht es etwa bei der Bedarfsplanung der Feuerwehr und der Orientierung der Stadtplanung am Konzept der Angsträume nicht allein um Zahlen, sondern ebenfalls um ihre Interpretation und Kontextualisierung, aus denen eine Vielzahl von (Sicherheits-)Erwartungen auch auf Seiten der Bürger*innen erwachsen. Krüger et al. stellen fest, dass Statistik und Imaginationen strukturierend auf die Praxis der Sicherheitsproduktion einwirken und kaum Raum für eine subjektive Perspektive auf Sicherheit lassen. Umgekehrt muss jedoch auch danach gefragt werden, ob und inwieweit das subjektive Sicherheitsempfinden überhaupt zu einer Handlungsmaxime der Sicherheitsproduktion gemacht werden sollte (vgl. Ammicht Quinn et al. 2017, S. 18; Görgen und Kraus 2013).

Bedrohungen des subjektiven Sicherheitsgefühls bilden häufig den Ausgangspunkt zivilgesellschaftlichen Sicherheitshandelns, dessen Ambivalenzen der abschließende Beitrag von Peter Bescherer adressiert. Während wissenschaftliche Beobachter*innen und politische Öffentlichkeit geradezu reflexhaft mit überwiegender Skepsis auf bürgerschaftliches Engagement im Bereich der Sicherheitsproduktion reagieren, argumentiert Bescherer, dass die vielfältigen Ansprüche auf Sicherheit nicht zwangsläufig in Ausgrenzung und normalisierender Sozialkontrolle münden müssen. Auf der Basis leitfadengestützter Interviews und teilnehmender Beobachtungen von zivilgesellschaftlichen (Sicherheits-)Akteur*innen in zwei deutschen Großstädten zeigt er die unterschiedlichen Motive und Gegenstände ihres Handelns auf, die sich in einem Spannungsfeld von Ver- und Entsicherheitlichung bewegen. Zwar deutet das empirische Material darauf hin, dass Bürger*innen ,Sicherheit ${ }^{*}$ keineswegs als oberste Priorität verstehen, wenn sie sich in lokalen Zusammenhängen für mehr Sicherheit engagieren. Die Möglichkeit einer - ebenfalls nicht auszuschließenden - Versicherheitlichung sozialer und politischer Fragen aber lässt die Forderung nach einer stärkeren Bürger*innenbeteiligung in der kommunalen Sicherheitsarbeit nur begrenzt sinnvoll erscheinen. Bescherer betrachtet bürgerschaftliches Engagement als Bestandteil eines umfassenden Demokratisierungsprozesses, in dem die Partizipation für Sicherheit nur dann inklusiv und solidarisch sein kann, wenn Beteiligungsverfahren auch bei sozial- und wirtschaftspolitischen Entscheidungen gestärkt werden.

Danksagung Das vorliegende Schwerpunktheft wäre ohne die Unterstützung verschiedener Kolleg*innen nicht möglich gewesen. Wir möchten uns an dieser Stelle zunächst sehr herzlich bei den Autor*innen bedanken, die das Heft nicht nur mit ihren Beiträgen bereichert haben, sondern die auch die Anregungen der Reviewer*innen und Herausgeber aufgegriffen und ihre Manuskripte auch unter größerem Zeitdruck umgehend überarbeitet haben. Unser Dank gilt darüber hinaus den Reviewer*innen, die ungeachtet ihrer Einbindung in die vielfältigen Geschäfte des Alltags bereit waren, die Beiträge kritisch und konstruktiv zu kommentieren. Axel Groenemeyer hat das Vorhaben dieses Schwerpunktheftes frühzeitig ermöglicht und auch nach dem Wechsel der Redaktion wurden wir durch Mechthild Bereswill und Anke Neuber in vielfältiger Weise unterstützt. Auch dafür möchten wir uns sehr herzlich bedanken. 


\section{Literatur}

Ammicht Quinn, Regina, Peter Bescherer, Friedrich Gabel, und Alexander Krahmer. 2017. Leitlinien für eine gerechte Verteilung von Sicherheit in der Stadt. Tübingen: IZEW.

Burt, Ronald. 1992. Structural holes. The social structure of competition. Cambridge: Harvard University Press.

Eick, Volker. 2014. Ein pazifiziertes Berlin? Vom Lohn von Law and Order. In Reclaim Berlin. Soziale Kämpfe in der neoliberalen Stadt, Hrsg. A. Holm, 153-180. Berlin, Hamburg: Assoziation A.

Feest, Johannes, und Erhard Blankenburg. 1972. Die Definitionsmacht der Polizei. Strategien der Strafverfolgung und soziale Selektion. Düsseldorf: Bertelsmann.

Foucault, Michel. 2004. Sicherheit, Territorium, Bevölkerung. Vorlesung am Collège de France 1977-1978. Frankfurt am Main: Suhrkamp.

Görgen, Thomas, und Benjamin Kraus. 2013. Subjektives Sicherheitsempfinden als polizeiliche Handlungsmaxime? In Rechtspsychologie, Kriminologie und Praxis: Festschrift für Rudolf Egg zum 65. Geburtstag, Hrsg. A. Dessecker, W. Sohn, 223-246. Wiesbaden: Kriminologische Zentralstelle.

Hall, Stuart, Chas Critcher, Tony Jefferson, John Clarke, und Brian Roberts. 1978. Policing the crisis. Mugging, the state, and law and order. London, Basingstoke: Macmillan.

Kelling, George E., und James Q. Wilson. 1982. Broken windows: the police and neighborhood safety. Atlantic Monthly 249(3):29-38.

Lefebvre, Henri. 1991. The production of space. Oxford: Blackwell.

Putnam, Robert. 2007. E pluribus unum: diversity and community in the twenty-first century. The 2006 Johan Skytte prize lecture. Scandinavian Political Studies 30(2):137-174.

Sampson, Robert J. 2012. Great American city. Chicago and the enduring neighborhood effect. Chicago: University of Chicago Press.

Stieber, Benno. 2017. Chronik eines Shitstorms. die tageszeitung, 22./23. Juli 2017.

Tyler, Tom R. 1990. Why people obey the law: procedural justice, legitimacy, and compliance. New Haven: Yale University Press. 